\title{
Calidad de Emulsiones de Carne de Búfalo (bubalus bubalis) tratadas con Ultrasonido de Alta Intensidad
}

\author{
Ramiro Torres ${ }^{1 *}$, Pedro Romero ${ }^{2}$ y Víctor M. Gelvez ${ }^{3}$ \\ (1) Facultad de Ingeniería, Programa de Ingeniería Agroindustrial, Univ. del Atlántico, Carrera 30 Número 8- 49, \\ Puerto Colombia-Colombia (e-mail: ramirotorres@mail.uniatlantico.edu.co) \\ (2) Facultad de Ingeniería, Departamento de Ingeniería de Alimentos, Univ. de Córdoba, Carrera 6 No. 76-103 \\ Montería - Córdoba (e-mail: promero@correo.unicordoba.edu.co) \\ (3) Facultad de Ingenierías y Arquitectura, Departamento de Alimentos, Univ. de Pamplona, Km 1, \\ vía salida a Bucaramanga, (e-mail: vmgelevez@uinipamplona.edu.co)
}

Recibido Sep. 6, 2018; Aceptado Nov. 30, 2018; Versión final Dic. 20, 2018, Publicado Jun. 2019

\begin{abstract}
Resumen
Se determinó el efecto del ultrasonido a diferentes tiempos y temperatura sobre el $\mathrm{pH}$, la estabilidad de la emulsión y la textura de emulsiones preparadas con carne de búfalo. Se prepararon emulsiones, se trataron con ultrasonido a $37 \mathrm{kHz}$ durante 3, 5 y $10 \mathrm{~min}$, se escaldaron, empacaron al vacío y almacenaron a $4^{\circ} \mathrm{C}$. $\mathrm{La}$ calidad de la emulsión se evalúo midiendo el pH, la estabilidad de la emulsión y un análisis de perfil de textura. El pH de la emulsión preparada con carne de búfalo disminuye cuando es tratada con ultrasonido a 3 y 5 min mientras que a 10 min no presento diferencia significativa con el control (muestras no tratadas con ultrasonido). La estabilidad de la emulsión aumenta con el tratamiento de ultrasonido. La dureza y la masticabilidad disminuyeron significativamente (significancia de 5\%) respecto al control, mientras que la elasticidad y la cohesividad no fueron afectadas por el tratamiento.
\end{abstract}

\section{Quality of Buffalo (bubalus bubalis) Meat Emulsions Treated with High Power Ultrasound.}

\begin{abstract}
The effect of ultrasound at different times and temperatures on $\mathrm{pH}$, emulsion stability and texture of buffalo meat emulsions was determined. Emulsions were prepared, treated with ultrasound at $37 \mathrm{kHz}$ for 3,5 and 10 $\mathrm{min}$, blanched, vacuum packed and stored at $4^{\circ} \mathrm{C}$. Emulsion quality was evaluated by measuring $\mathrm{pH}$, emulsion stability and a texture profile analysis. The $\mathrm{pH}$ of the emulsion prepared with buffalo meat decreases when it is treated with ultrasound at 3 and $5 \mathrm{~min}$ while at $10 \mathrm{~min}$ it does not present a significant difference with the control (samples not treated with ultrasound). The stability of the emulsion increases with ultrasound treatment. Hardness and chewiness decreased significantly (significance of 5\%) with respect to control, while elasticity and cohesiveness were not affected by treatment.
\end{abstract}

Keywords: elasticity; meat emulsions; ultrasound; texture; chewiness 


\section{INTRODUCCIÓN}

El total de cabezas bubalinas el 2016 llegan a unas 199.3 millones en el mundo, la gran mayoría $(97,2 \%)$ se encuentran en el continente Asiático con unos 194 millones de cabezas. La India contribuye con el 58\%, Pakistán el 19\% y China continental el 12,3\%. En países de Europa, África y en América se encuentra el resto de la población (FAO, 2017). En América con excepción de Chile y Canadá, se estima que existen 4.09 millones de cabezas. Los países suramericanos con mayor población bubalina son Brasil 83.5\%, seguido de Argentina, Venezuela y Colombia (López, 2013). En Colombia, la existencia de grandes extensiones de tierras inundables en regiones caracterizadas por una marcada estacionalidad de lluvias, con suelos de mal drenaje y baja fertilidad, en las que el ganado vacuno presenta problemas para producir eficientemente, el búfalo de agua (Bubalus bubalis) se convierte en una especie con gran potencial para la producción de carne y leche de primera calidad. Estudios realizados demuestran que el búfalo tiene la capacidad de aprovechar con mayor eficiencia los forrajes de baja calidad y tener una mejor respuesta de adaptación al trópico que el ganado vacuno Bos indicus y Bos taurus reflejándose en ganancias de peso superiores y en la disminución de los parámetros reproductivos de importancia económica tales como la edad al servicio efectivo y el intervalo entre partos (Fraga et al., 2017).

Desde el 2010, en Colombia, se ha presentado un incremento promedio anual del $16 \%$ de cabezas bubalinas de manera sostenida hasta el 2017, donde se ha reportado una existencia de 651.000 animales, estableciéndose en la industria ganadera del país, debido a que producen carne y leche en condiciones donde el ganado vacuno puede escasamente sobrevivir (ACB, 2018). Gran parte de los rebaños se encuentran distribuidos en los departamentos de Córdoba, Antioquia, Santander, Caldas, Cesar, Cundinamarca, Valle, Cauca, zonas de Llanos Orientales y Amazonas. Probablemente su crecimiento se deba a que el precio de la leche de búfala es pagado un 30\% por encima de la leche de vaca, básicamente por su mayo contenido de sólidos totales, y que alcanzan el peso de sacrificio antes que el ganado vacuno indicando que es un animal precoz para producir carne con alta calidad fisicoquímica y organoléptica, por lo que el búfalo puede convertirse en un recurso cárnico de alta calidad (Li et al., 2018).

Estudios han reportado que la carne del búfalo es tan aceptable como la del ganado y que pueden retener sensibilidad de la carne a una edad más avanzada que la del ganado debido a que el tejido conectivo se endurece más tarde, mejorando la calidad de la carne de búfalo (Uriyapongson, 2013). La carne de búfalo a la edad apropiada de sacrificio es más tierna y fresca que la carne de vaca, rica en proteínas, aminoácidos esenciales altos, con baja grasa intramuscular, ácidos grasos saturados bajos, con menor contenido de colesterol $(56.9 \mathrm{mg} / 100 \mathrm{~g}$ vs $106.6 \mathrm{mg} / 100 \mathrm{~g})$ y triglicéridos $(1,38 \mathrm{~g} / 100 \mathrm{~g}$ vs $1,77 \mathrm{~g} / 100 \mathrm{~g})$ en comparación con la carne vacuna. Además, la carne de búfalo tiene más ácidos grasos esenciales como el omega 6 y el omega 3 ( 6.12 y $2.77 \%$ del total de ácidos grasos), los cuales están estrechamente relacionado con la salud humana. Aunque contiene menor cantidad de Omega 6 que el cerdo y el pollo, el contenido de Omega 3 es más alto. Tiene alto contenido de ácido eicosapentaenoico (EPA) y El ácido docosahexaenoico (DHA) comparada con la de ternera y cerdo los cuales son importantes para el desarrollo del cerebro. (Li et al., 2018; Uriyapongson, 2013).

La calidad organoléptica de la carne de búfalo después de las 36 semanas de edad es alta y presenta mejores propiedades fisicoquímicos son más altos. Sin embargo, este presenta sus mejores características de calidad después de 2 años. Aunque el porcentaje de carne útil disminuye con la edad (54.93\% a 51.22\%), el porcentaje de carne y el rendimiento de la carne en canal aumentaron gradualmente (el espesor de la grasa dorsal y el área del costillar) lo que le convierte al búfalo en un recurso cárnico de alta calidad (Li et al., 2018). La calidad de la carne depende del aroma, sabor, apariencia, textura y jugosidad. El comportamiento del consumidor indica que la textura es el factor de palatabilidad más importante para determinar la calidad de la carne. Sin embargo, la textura depende de factores tales como la terneza de la carne, su capacidad de retención de agua (CRA) relacionada con la jugosidad y también el grado de maduración (Alarcón et al., 2015). Aunque la textura es la propiedad sensorial de los alimentos que es detectada por los sentidos del tacto, la vista, el oído, y que se manifiesta cuando el alimento sufre una deformación. No se puede hablar de la textura de un alimento como una propiedad única de éste, sino que hay que referirse a los atributos o a las propiedades de textura de ese alimento.

Las principales características de la textura, que se pueden obtener mediante el análisis del perfil de textura (TPA), son: dureza $(\mathrm{N})$ que es la fuerza máxima requerida para comprimir un alimento ente los molares o fuerza para deformar al alimento, la adhesividad $(\mathrm{N} \times \mathrm{mm})$, área debajo de la abscisa después de la primera compresión, que representa la energía requerida para superar la tracción entre la superficie del alimento y la boca, cohesividad que es la medida en que la muestra podría deformarse antes de la ruptura, elasticidad que es la capacidad de la muestra para recuperar su forma original después de haber eliminado la fuerza de deformación y la masticabilidad $(\mathrm{N})$ que es I fuerza necesaria para masticar un alimento solido hasta un estado tal que permita su ingesta ( Rosenthal, 1999) 
El uso de ultrasonido como tecnología emergente para procesamiento de alimentos ha sido motivo de investigación y desarrollo desde hace muchos años (Robles y Ochoa 2012; Bermúdez et al., 2011). En ultrasonido es una tecnología innovadora que tiene aplicaciones tanto en el análisis como el procesamiento de alimentos, y en particular la aplicación de ondas ultrasónicas a las carne o emulsiones cárnicas puede inducir alteraciones mecánicas de las estructuras intracelulares y mejorar su textura. Además, el tratamiento con ultrasonido tiene la ventaja de ser no invasivo (Kang, et al., 2016; Got et al., 1999). El ultrasonido se define como una forma de energía que viaja en ondas de sonido iguales o superiores a $20 \mathrm{kHz}$, que, al trasportarse a través de un medio, promueve la formación de burbujas microscópicas que oscilan y crecen en tamaño hasta que la burbuja implosione, o colapso, dando como resultado el fenómeno conocido como cavitación, provocando altas temperaturas y presiones. La eficiencia de la cavitación depende de varios factores. Sin embargo, predominantemente la frecuencia de la onda de sonido determinará si se produce cavitación y si ésta es estable o transitoria. A frecuencias de $18-100 \mathrm{kHz}$, las burbujas microscópicas de gas tienen aproximadamente $25 \mu$ s para crecer durante el ciclo de rarefacción, lo que conduce a una cavitación menos frecuentes pero más violentas que a frecuencias mayores a $1 \mathrm{MHz}$, donde las microburbujas tienen solo aproximadamente $0,5 \mu \mathrm{s}$ para crecer. Si este colapso se da dentro de un material biológico, el ultrasonido puede afectar los tejidos biológicos a micro y macroescala. En el caso del procesamiento de alimentos, los efectos son, en general, positivos ya que se pueden aplicar para promover una mayor calidad e inocuidad de los alimentos (Warner. et al, 2017; Chandrapala et al., 2012).

Según los rangos de sonido se dividen en ultrasonido de alta frecuencia y baja intensidad ( $1 \mathrm{MHz},<1 \mathrm{~W}$ $\mathrm{cm}^{-2}$ ) que no es destructivo y se utiliza para el análisis o caracterización de compuestos, y ultrasonido de baja frecuencia y alta intensidad (20 a $100 \mathrm{kHz}$ con 10-1000 W cm -2), también conocido como ultrasonido de potencia. En el procesamiento de alimentos, el ultrasonido de alta intensidad a bajas frecuencias, de 20 a $100 \mathrm{kHz}$, es útil para inactivar microorganismos, extender su vida útil y operaciones de procesamiento de alimentos como ablandamiento, marinado, descongelación, liofilización y concentración, secado, extracción de aromatizantes y mejorar las propiedades de la carne (Warner. et al, 2017).). El uso del ultrasonido es una técnica prometedora en la tecnología alimentaria, dado su incidencia del sobre las propiedades funcionales de diferentes fuentes de proteínas y su bajo impacto en el medio ambiente, convirtiéndose en una tecnología ecológica En los últimos años, el ultrasonido de alta intensidad ha sido utilizado como una alternativa para mejorar las propiedades funcionales de proteínas de varias fuentes animal como vegetal. Investigadores han reportado efectos significativos del tratamiento de ultrasonido sobre la formación de espuma, solubilidad, emulsión y otras propiedades funcionales de estas proteínas. Los efectos de la implementación del tratamiento sobre estas propiedades dependen de las características inherentes de la fuente de proteína y de la intensidad y amplitud del ultrasonido, así como en el pH, la temperatura, la fuerza iónica, el tiempo, y todas las variables que tienen un efecto sobre las propiedades fisicoquímicas de las proteínas, por lo que es necesario establecer las mejores condiciones de tratamiento o las condiciones de optimización del proceso para cada tipo de alimento. (Higuera, et al 2016).

También se ha reportado estudios donde se ha utilizado el tratamiento, de ultrasonido para mejoramiento de las características sensoriales, específicamente las texturales como terneza, en diferentes tipos de músculos vacuna (Barekat y Soltanizadeh, 2017) y aves (Zuo et al 2018). Se ha sugerido que el ablandamiento de la carne post-rigor usando ultrasonido es por uno o una combinación de; (a) disrupción física del tejido causada por cavitación (b) liberación y activación de enzimas y / o por (c) alterar el metabolismo en la carne previa al rigor mediante la liberación de calcio. La aplicación de ultrasonido tiene cierto potencial para mejorar la textura de la carne post rigor. Sin embargo, para que esto sea una viabilidad comercial, las condiciones acústicas deben ser investigadas a fondo para optimizar las condiciones de ablandamiento (Warner. et al, 2017).

En Colombia, el conocimiento de la producción de bovinos para carne se limita a registrar algunos parámetros de desarrollo de los animales en pie, tales como la ganancia de peso diario, peso al nacimiento, peso al destete, el peso vivo al sacrificio y eventualmente algunos datos sobre las canales, sin embargo, son pocos los trabajos publicados que describen las características de calidad de las canales, y emulsiones procesadas con tecnologías emergentes. Por tal razón, el objetivo del presente trabajo fue evaluar el efecto del ultrasonido $(37 \mathrm{kHz})$ en función del tiempo $(60,120,240 \mathrm{~s})$ a temperatura de $30^{\circ} \mathrm{C}$, sobre el pH, el EE y la textura, de emulsiones preparadas con carne de búfalo.

\section{METODOLOGÍA}

La carne de Búfalo (bubalus bubalis) en cortes de primera calidad, obtenida en el mercado local de la ciudad de Montería (Colombia) se adecuó retirando partes indeseables (grasa y tendones) y se cortó en cubos (2 $\mathrm{cm}$ ); se sometió a molienda empleando un molino (Ortega № 22) con el disco de diámetro № 6 . 


\section{Preparación de la emulsión y condicionamiento}

La preparación de la emulsión se realizó según la fórmula propuesta por Sachindra et al. (2005), se modificó el porcentaje de carne (a 69.62\%), se reemplazó la grasa de porcino por aceite vegetal. La carne molida, aceite vegetal, hielo, fosfato, almidón, sal curante y sal común se sometieron a emulsificación en un cutter (BlakesLee) de $3 \mathrm{~L}$. La emulsión se porcionó ( $20 \mathrm{~g} / \mathrm{unidad}$ ) en tripa sintética de celulosa calibre 22, empleando una embutidora hidráulica (RAMON, de 15 L), se empacó al vacío (90\%) en una empacadora (Henkovac 1500) y se almacenó a $4^{\circ} \mathrm{C}$.

\section{Aplicación de US de alta intensidad}

Las muestras empacadas al vacío se trataron durante 3, 5 y 10 minutos en un baño de ultrasonido (Elmasonic $\mathrm{E}-60$ ) con una frecuencia de $37 \mathrm{kHz}$ y $25 \mathrm{~W} / \mathrm{cm}^{2}$. Como medio de difusión se empleó agua desionizada (5 I), a $16^{\circ} \mathrm{C}$. Todas las muestras tratadas se almacenaron a $2^{\circ} \mathrm{C}$. Se dejaron muestras sin tratar como control. Las muestras de emulsión tratadas con US, se escaldaron con agua caliente $\left(72^{\circ} \mathrm{C}\right)$ hasta alcanzar una temperatura interna $\left(70^{\circ} \mathrm{C}\right)$ durante 18 minutos. Se enfriaron, se empacaron al vacío $(90 \%)$ en una empacadora (Henkovack) y se almacenaron a $4^{\circ} \mathrm{C}$.

\section{pH y estabilidad de la emulsión}

Se determinó el pH, de emulsión de carne de búfalo, por lectura directa, utilizando un pH-metro digital (pH meter 744, Metrohm). La estabilidad de la emulsión (EE) tratada con ultrasonido en los diferentes tiempos (3, 5 y $10 \mathrm{~min}$ ) y sin tratae, se determinó según procedimiento de Thomas et al. (2006). Se tomaron veinticinco gramos de la emulsión / masa de carne en una bolsa de Polietileno de baja densidad (LDPE) y se calentaron en un baño de agua controlado por termostato a $80^{\circ} \mathrm{C}$ durante $20 \mathrm{~min}$. La estabilidad se calculó a partir de la pérdida de peso durante la cocción y se expresó como porcentaje del peso inicial.

\section{Análisis de Perfil de Textura (TPA)}

El análisis del perfil de textura de emulsión de carne de búfalo, se llevó a cabo según el procedimiento descrito por Torres et al., (2015), utilizando un analizador de textura TA-XT plus de Stable Microsystems Texturometer (Stable Microsystems Ltd. Surrey, Inglaterra, Reino Unido), programado y controlado por el software Nexigen ®. Las muestras enfriadas se atemperaron a temperatura ambiente $\left(27 \pm 0,5^{\circ} \mathrm{C}\right)$. Se utilizaron muestras cilíndricas, de $2 \mathrm{~cm}$ de diámetro y $2 \mathrm{~cm}$ de atura, las cuales se sometieron a una doble compresión unidireccional, utilizando una celda de compresión cilíndrica de $10 \mathrm{~cm}$ de diámetro y una celda de carga 25 $\mathrm{kg}$, hasta el $40 \%$ de su altura original, a una velocidad de celda de $50 \mathrm{~mm} / \mathrm{min}$, dejando transcurrir un tiempo de $5 \mathrm{~s}$ entre los dos ciclos de compresión. Los parámetros determinados de las curvas obtenidas de deformación fuerza fueron: dureza $(\mathrm{N})$ : fuerza máxima / energía requerida para comprimir la muestra; Cohesividad: medida en que la muestra podría deformarse antes de la ruptura (A2 / A1, A1 es la fuerza máxima requerida para la primera compresión y A2 es la fuerza máxima requerida para la segunda compresión); elasticidad $(\mathrm{mm})$ : altura que la muestra recupera durante el tiempo que transcurre entre el final de la primera mordida y el inicio de la segunda mordida o la muestra de distancia recuperada después de la primera compresión, es decir, la capacidad de la muestra para recuperar su altura original después del se eliminó la fuerza de deformación; masticabilidad ( $\mathrm{N} \mathrm{mm}$ ) - trabajo requerido para masticar las muestras para la deglución (elasticidad $\times$ gomosidad).

\section{Diseño experimental y Análisis estadísticos}

El experimento se condujo bajo un diseño completamente al azar con un solo factor (tiempo de exposición a ultrasonido) en 4 niveles (0, 3, 5 y $10 \mathrm{~min})$, con 6 repeticiones para un total de 24 unidades experimentales. Se realizó un análisis de varianza (ANOVA) y prueba de diferencias mínimas significativa a un nivel de significancia del $5 \%$, para las variables de respuesta $\mathrm{pH}$, estabilidad de la emulsión (EE) y los parámetros texturales. El análisis de los datos se realizó con software Statistica 7.0 versión de prueba 20.

\section{RESULTADOS Y DISCUSIÓN}

En la fig 1 se observa el efecto de la aplicación de ultra sonido $(37 \mathrm{kHz}, 16 \stackrel{\circ}{\circ})$ ), sobre el pH y la estabilidad de la emulsión de Carne de Búfalo (bubalus bubalis). El pH de la muestra control es significativamente más alto que en muestras tratadas con ultrasonido a 3 y 5 min (tabla 1), mientras que con el valor del $\mathrm{pH}$ de la muestra tratada con ultrasonido a 10 min no presenta diferencia significativa (significancia del $5 \%$ ). El efecto del tratamiento con ultrasonido a 3 y 5 min sobre el $\mathrm{pH}$ puede ser el resultado del efecto de las altas presiones y temperaturas en la cavitación, permitiendo la generación de radicales libres (hidrogeniones), cambios en la estructura helicoidal de la proteína y descomposición del agua, con lo que se aumenta la concentración de 
iones hidronio y por tanto el pH se disminuye (O'Sullivan, et al., 2016;) El comportamiento de las muestras tratadas a los $10 \mathrm{~min}$ (aumento del valor del pH respecto a las tratadas a 3 y $5 \mathrm{~min}$ ) es causado por (lisis celular) provocando la liberación de iones del interior de la célula en el citoplasma y un cambio en la estructura de la proteína. Estos resultados concuerdan con lo reportado con lo reportado por Fuentes et al. (2016) quienes sometieron a tratamiento con ultrasonido $(37 \mathrm{kHz}, 25 \mathrm{~W} . \mathrm{cm}-2$ durante $0,3,4$ y $5 \mathrm{~min})$ carne de Lomo Atún (Thunnus albacares) con lo cual se afectó el pH con relación a la muestra control a los 4 y 5 min de exposición.

La disminución del pH se asoció con el contenido de glucógeno en el momento del sacrificio. Las condiciones de estrés podrían reducir el contenido de glucógeno en los músculos. Los niveles de glucógeno podrían llevar a una glucólisis baja y luego a una leve de ácido láctico, por lo que la disminución del pH también fue baja. El valor final del pH no sólo se vio influenciado por el glucógeno. Aberle y otros (2001) sugirieron que los cambios en el $\mathrm{pH}$ de la carne de vacuno se veían afectados de forma muy significativa por factores intrínsecos como las especies, el tipo de músculo, los animales individuales y por factores extrínsecos como la manipulación del ganado antes y después del sacrificio. Aunque algunos autores como Higuera et al. (2017) han reportado en proteínas de calamar (Dosidicus gigas) que el ultrasonido no afecta el pH considerando que los tratamientos de ultrasonido no afectan la ionización de los grupos ionizables de las proteínas, lo que significa que las condiciones de tratamiento con ultrasonido $(20 \mathrm{kHz}$ a diferentes niveles de amplitud $(0,20$ y $40 \%)$ durante $0,30,60$ y $90 \mathrm{~s}$.) no promueven la protonación o la hidrólisis de proteínas. De igual manera Hu et al (2014) reportaron similares resultados al aplicar tratamiento con ultrasonido (20 a $140 \mathrm{kHz}$ en de 10 a $50 \mathrm{~min}$ ) a porciones de calamar gigante (Dosidicus gigas), sin encontrar diferencias significativas (significancia de 5\%) en los cambios de $\mathrm{pH}$.

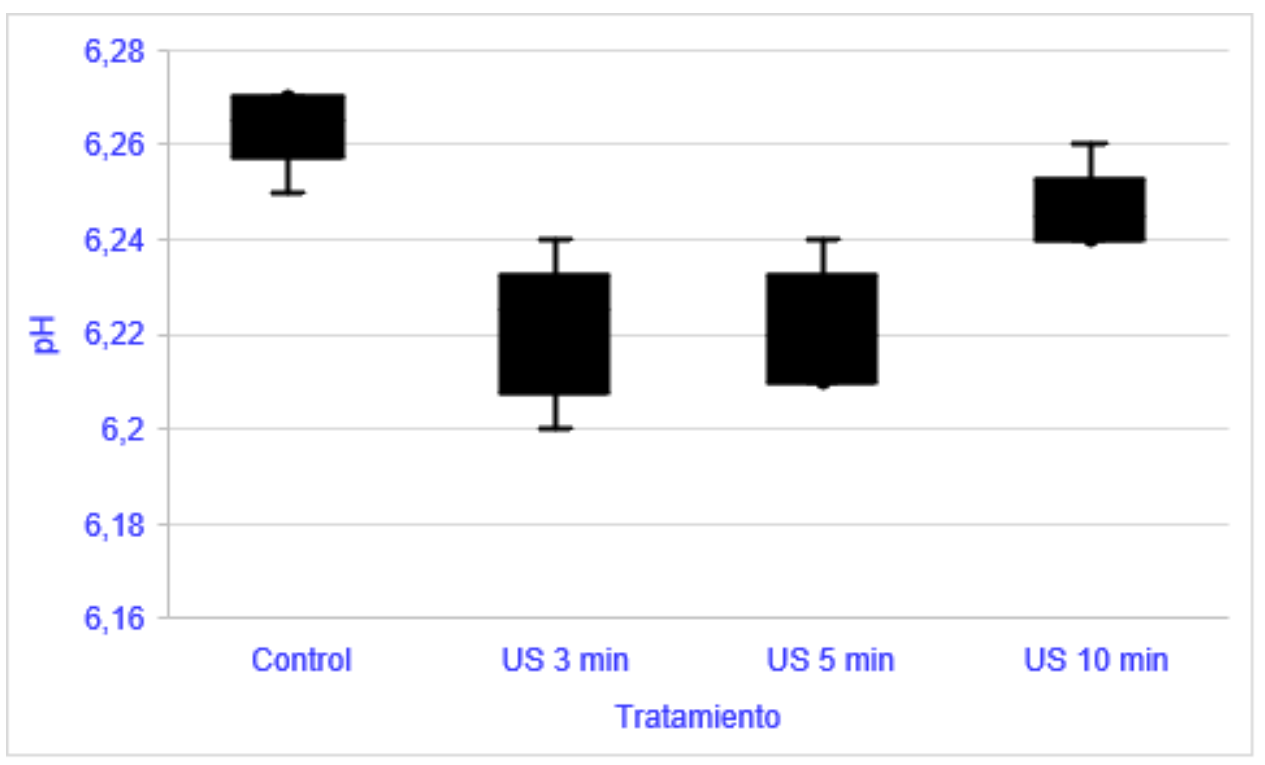

Fig. 1: Efecto del ultrasonido $\left(37 \mathrm{kHz}, 16^{\circ} \mathrm{C}\right)$ sobre el $\mathrm{pH}$ de la emulsión de carne búfalo.

Sikes et al 2014, por su parte, al aplicar ultrasonido de alta frecuencia al músculo pre-rigor mortis, imitando la estimulación eléctrica, con el propósito de acelerar la glucólisis reportaron que la aplicación del ultrasonido (2 $\mathrm{MHz}$, ajuste la potencia $60 \%$ a $30^{\circ} \mathrm{C}$ y con un tiempo de exposición de $10 \mathrm{~min}$ ) no tuvo un efecto significativo (significancia del $5 \%$ ) sobre el $\mathrm{pH}$ de pretratamiento y postratamiento de la carne de vacuno pre-rigor mortis. En este mismo sentido, sin embargo, Got et al. (1999) aplicaron ultrasonido de alta frecuencia y alta frecuencia (2.6 $\mathrm{MHz}, 10 \mathrm{~W} . \mathrm{cm}^{-2}$ durante $15 \mathrm{~s}$ ) para pre-rigor de la carne de vacuno y encontraron un ligero retraso en el inicio del rigor mortis aunque el valor de $\mathrm{pH}$ final fue en promedio de 5.4 y no difirió significativamente entre las muestras de control y las tratadas con ultrasonido (significación de $5 \%$ ). Los autores sugieren que retraso inicial en la acidificación de los músculos tratados podría deberse más al aumento del pH inducido por el tratamiento con ultrasonido, el cual acelera la liberación de iones desde la estructura celular al citosol, dado que inmediatamente después de la aplicación de ultrasonido $(\mathrm{pH} 6,2)$, el $\mathrm{Ca}^{++}$libre, en el espacio intermofibrilar, aumentó en un 30\% ( significación de $5 \%$ ) en comparación con los controles fijados al mismo $\mathrm{pH}$ post-mortem, lo que sugiere una gran liberación de estos iones en el citosol o a un cambio en la estructura de la proteína que provocó una modificación en la posición de algunos grupos iónicos

Según Higuera et al. (2017), los cambios de pH dependen de varios factores de las carne y emulsiones tratadas con ultrasonido dependen de las condiciones del tratamiento (frecuencia, tiempo, amplitud, potencia), así como las condiciones de la muestra, como la concentración de proteínas, la geometría de la muestra o 
las dimensiones. Por lo que es muy importante definir las condiciones óptimas para desnaturalizar proteínas, pero sin causar su hidrólisis. El efecto del tratamiento sobre la estabilidad de la emulsión (EE) de carne de búfalo (Bubalus bubalis), se observa un aumento significativo (grado de significancia del $5 \%$ ) entre las muestras tratadas con ultrasonido y las muestras no tratada (fig.2), aunque el tiempo de exposición no influye significativamente en la estabilidad de la emulsión. El aumento de la estabilidad de la emulsión conseguida con el tratamiento, es debido a una adecuada formación de película proteica interfacial constituida por proteínas solubles, actomiosina y miosina, liberadas por el rompimiento de las ondas ultrasónicas, influyendo en la capacidad de ligazón. Por otra parte, la presión de cavitación permite disminuir el tamaño de las partículas favoreciendo la agregación y permitiendo además estabilizar los glóbulos grasos para evitar la coalescencia (Zhang et al., 2014).

Estos resultados concuerdan con lo reportado por O'Sullivan, et al. (2005), donde se ha reportado que la solubilidad de concentrado de proteína vegetales y el rendimiento emulsionante de proteína animal, proteína de clara de huevo y concentrado de proteína de arveja mejoraron después del tratamiento con ultrasonidos (20 kHz, intensidad acústica de $34 \mathrm{~W} / \mathrm{cm} 2$ durante $2 \mathrm{~min}$ ). Los autores consideran que la mejora en la estabilidad de la emulsión tratadas con ultrasonido, está asociada con un aumento en la hidrofobicidad (es decir, disminución de la viscosidad intrínseca) y un mejor empaquetamiento interfacial observa por una disminución en la tensión interfacial de equilibrio y aunque el con ultrasonido redujo el tamaño de las proteínas, y no observaron ninguna reducción en el peso molecular de la estructura primaria de éstas.

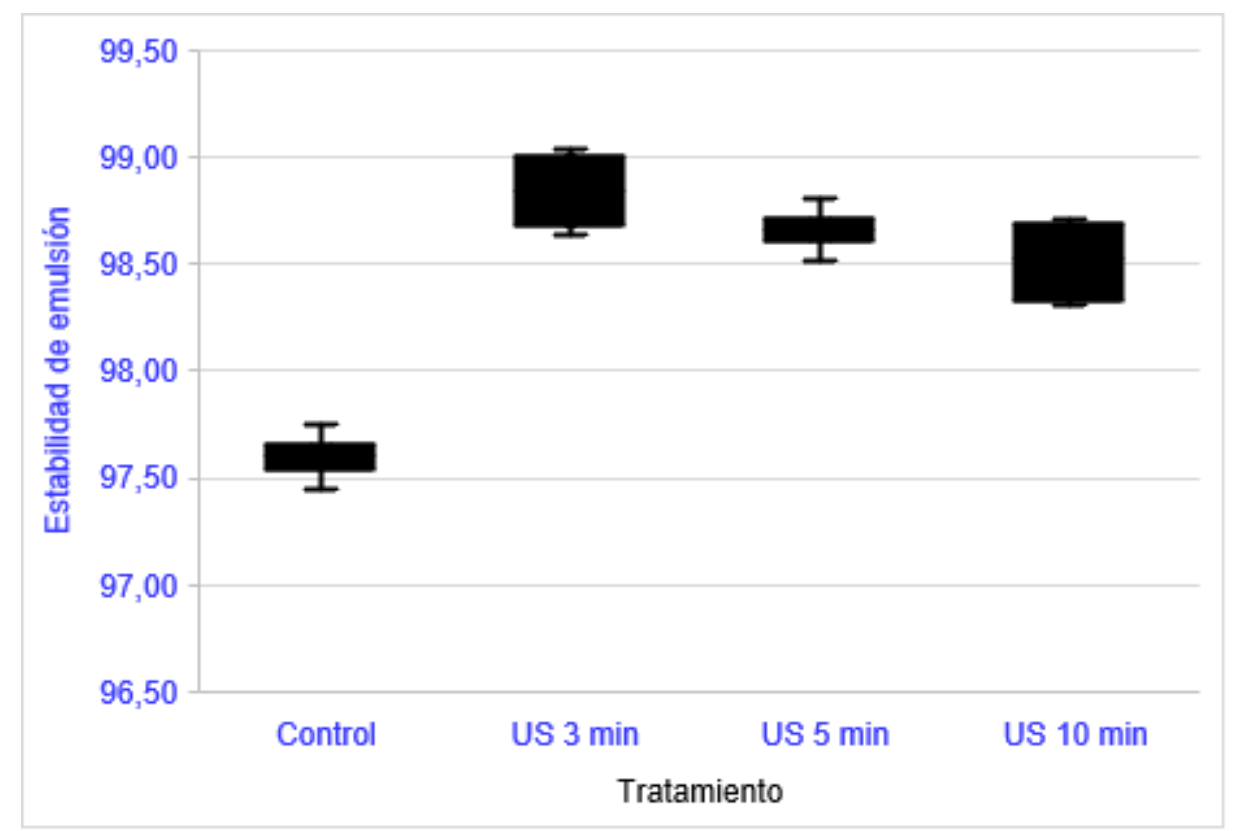

Fig. 2: Efecto del ultrasonido $\left(37 \mathrm{kHz}, 16^{\circ} \mathrm{C}\right)$ sobre la estabilidad de la emulsión de carne búfalo.

Las propiedades de retención de agua y grasa de los productos cárnicos triturados, como las emulsiones cárnicas, dependen vitalmente de la formación de una matriz proteica (La matriz de gel de las interacciones proteína-proteína tiene la capacidad de unirse al agua y contener glóbulos de grasa. Entre las proteínas cárnicas, las proteínas miofibrilares que se extraen en la fase acuosa durante la trituración y la mezcla generalmente se consideran el factor más importante para la emulsificación y la calidad de la red de carne, debido a su carácter anfifílico, forma una película monocapa superficial en la interfase de grasa agua con su parte hidrófila hacia el agua y su parte hidrófoba orientada hacia grasa. El tratamiento con ultrasonido independiente del tiempo de exposición podría favorecer la formación una red de proteínas miofibrilares densa, que retiene más eficientemente el agua y la grasa dentro de la matriz de proteínas, actuando como emulsionantes adsorbiéndose preferiblemente a la interfaz agua-grasa (Sorapukdee et al., 2013).

\section{Análisis de perfil de textura (TPA)}

Como se muestra en la Tabla 1, hay una reducción progresiva (significancia del $5 \%$ ) en la dureza y masticabilidad de la emulsión de carne de búfalo con el aumento del tiempo de exposición del tratamiento con ultrasonido. Esta reducción en la dureza podría deberse a los mayores gradientes de temperatura en el sitio de colapso de burbujas. Esto se debe a que el aumento de la temperatura conduce a una reducción de la dureza debido a la desnaturalización de las proteínas, algo similar a la carne pálida, blanda y exudativa (Jayasooriya et al., 2007). 
Estos resultados concuerdan con los reportados por Zou et al (2018) para la fuerza de corte de la carne de pechuga de ganso tardada con ultrasonido $(20 \mathrm{kHz}$ a $800 \mathrm{~W}$ durante $20 \mathrm{~min})$. Los autores consideran que la disminución de la fuerza de corte para las muestras podría deberse al efecto de ultrasonido sobre la integridad y compactibilidad de miofibrillas y la capacidad de retención de agua de las carnes y consideran que el tratamiento ultrasónico puede lograr un mejor efecto de ablandamiento que ningún tratamiento en un corto tiempo. Dado que el valor de la fuerza de corte está estrechamente relacionado con el diámetro y la madurez de la fibra muscular, y el contenido de humedad, ésta es un indicador directo de la sensibilidad muscular, donde un valor de fuerza de corte bajo indica una mejor terneza de la carne. Por su parte La explicación de los valores reducidos de masticabilidad se pueden explicar por la mayor capacidad de retención de agua (CRA) de las muestras experimentales que según Singh, et al., (2013) ésta correlacionada con el contenido de humedad.

Tabla 1: Efecto del ultrasonido $\left(37 \mathrm{kHz}, 16^{\circ} \mathrm{C}\right)$ sobre la textura en una emulsión de carne de búfalo. Valores medios con letras diferentes presentan diferencias significativas $(P<0.05)$

\begin{tabular}{|c|c|c|c|c|}
\hline Tratamiento & Dureza $(N)$ & Cohesividad & Elasticidad & Masticabilidad (N) \\
\hline Control & $12.43 \pm 0.32^{\mathrm{a}}$ & $0.45 \pm 0.05^{\mathrm{a}}$ & $6.92 \pm 0.06^{\mathrm{a}}$ & $38.71 \pm 2.21 \mathrm{a}$ \\
\hline US 3min & $12.21 \pm 0.25^{\mathrm{a}}$ & $0.48 \pm 0.04^{\mathrm{a}}$ & $6.48 \pm 0.20^{\mathrm{a}}$ & $37.98 \pm 3.10^{\mathrm{b}}$ \\
\hline US 5 min & $11.40 \pm 0.82^{\mathrm{b}}$ & $0.49 \pm 0.02^{\mathrm{a}}$ & $6.02 \pm 0.08^{\mathrm{a}}$ & $33.63 \pm 2.22^{\mathrm{b}}$ \\
\hline US 10 min & $10.50 \pm 0.31^{\mathrm{c}}$ & $0.44 \pm 0.04^{\mathrm{a}}$ & $6.71 \pm 0.17^{\mathrm{a}}$ & $31.00 \pm 3.40^{\mathrm{a}}$ \\
\hline
\end{tabular}

Las fuerzas hidrodinámicas y las altas temperaturas locales generadas por la cavitación inducida por ultrasonidos conducen a cambios en la estructura de los alimentos ( O'Sullivan et al., 2016 ). Según Jayasooriya et al., (2007) el fenómeno de la cavitación es responsable del debilitamiento físico de la estructura del alimento, los daños celulares y subcelulares, una distribución uniforme de los glóbulos grasos, mayor hidratación de la proteína y una reducción de los cambios de la estructura de la proteína, lo cual conduce a una reducción de la dureza de la carne. Resultados similares han sido reportados por Barekat y Soltanizadeh (2017) quienes reportaron una disminución de la dureza de la carne al aplicar tratamiento de ultrasonido (20 $\mathrm{kHz}, 400 \mathrm{~W}$ durante 10 a $30 \mathrm{~min}$ ) para músculos de carne bovina donde también afirman que la influencia del ultrasonido en la dureza depende de su duración y potencia, evidenciándose por la dureza reducida de 15.023 $\mathrm{N}$ a $3.969 \mathrm{~N}$ con el aumento de la duración del tratamiento con ultrasonidos (de 0 min a $30 \mathrm{~min}$ ) y la potencia (de 100 a $300 \mathrm{~W}$ ). Fuentes et al. (2016) retorta también una disminución de la dureza y masticabilidad de la carne de Lomo Atún (Thunnus albacares) al ser tarada con ultrasonido (37kHz, $400 \mathrm{~W}$ durante 0, 3, 4 y $5 \mathrm{~min}$ ).

Sin embargo estos resultados contrastan con los de Zhao et al., (2014) quienes estudiaron el efecto del US sobre las propiedades funcionales de gel compuesto de proteína miofibrilar de pechuga de pollo en el cual todas las muestras tratadas con ultrasonido $(20 \mathrm{kHz}$ a $450 \mathrm{~W}$, durante 3 a $12 \mathrm{~min}$ ) presentaron mayor dureza la masticabilidad que el control, lo cual explican por una mayor formación de interacción caseinato-proteínas miofibrilares dando como resultado una estructura de gel más fuerte durante el calentamiento. El tiempo de exposición del tratamiento con ultrasonido no tuvo ningún efecto significativo (significancia del $5 \%$ ) sobre la cohesividad y elasticidad la emulsión de carne de búfalo. La cohesión representa la fortaleza de los vínculos internos (Ruiz de Huidobro et al., 2005). Por lo tanto, la cohesión reducida se puede atribuir a la ruptura de enlaces débiles entre proteínas, lo que conduce a su solubilización

\section{CONCLUSIONES}

El pH de la emulsión preparada con carne industrial de búfalo disminuye cuando es tratada con ultrasonido $(37 \mathrm{kHz}, 16 \stackrel{\circ}{\circ}$ ) durante 3 y 5 mientras que a 10 min no presento diferencia con las muestras no tratada. La estabilidad de la emulsión de la emulsión preparada con carne industrial de búfalo aumentó con el tratamiento por ultrasonido sin embargo el tiempo de exposición no afectó la estabilidad. Las propiedades texturales de las emulsiones preparadas con carne de búfalo son afectas por el tratamiento con ultrasonido $\left(37 \mathrm{kHz}, 16^{\circ} \mathrm{C}\right)$. La dureza y la masticabilidad aumentan significativamente (significancia de $5 \%$ ) a mayor tiempo de tratamiento, mientras que la elasticidad y la cohesividad no son afectadas por el tratamiento.

\section{REFERENCIAS}

Alarcón, A.D., H. Janacua, J.C. Rodríguez, L. Paniwnyk y T.J, Mason, Power ultrasound in meat processing, doi: https://doi.org/10.1016/j.meatsci.2015.04.015, Meat Science, 107, 86-93 (2015)

ACB, El bufalo: Datos productivos, Asociación Colombiana de Criadores de Búfalos, Colombia (2017) 
Barekat, S. y N. Soltanizadeh, Improvement of meat tenderness by simultaneous application of high-intensity ultrasonic radiation and papain treatment, doi: https://doi.org/10.1016/j.ifset.2016.12.009, Innovative Food Science and Emerging Technologies, 39, 223-229 (2017)

Bermúdez, D., T. Mobbs y G. Barbosa-Cánovas, Ultrasound Applications in Food Processing. in: Ultrasound Technologies for Food and Bioprocessing by H. Feng, G. Barbosa-Cánovas G y J. Weiss, 66-105, Springer, New York, USA (2011)

Chandrapala, J., C. Oliver, S. Kentish y M. Ashokkumar, Ultrasonics in food processing - food quality assurance and food safety, doi: https://doi.org/10.1016/j.tifs.2012.01.010, Trends Food Sci. Technol., 26, 88-98 (2012)

FAO, Estadísticas Ganadería, FAOSTAT, USA (2017)

Fraga, L.M., D. García y otros siete autores, Productive and reproductive performance of water buffaloes in Empresa Pecuaria Genética del Este, in Cuba. Suggestions for their improvement, ISSN: 2079-3480, Cuban, Journal of Agricultural Science, 51(2), 1-7 (2017)

Fuentes, L., D. Acevedo y V. M. Gélvez, Efecto del Ultrasonido y Campos Magnéticos en la Carne de Lomo Atún (Thunnus albacares), doi: http://dx.doi.org/10.4067/S0718-07642016000200004, Información Tecnológica, 27(2), 21-30 (2016)

Got, F., J. Culioli y otros cinco autores, Effects of high-intensity high-frequency ultrasound on ageing rate, ultrastructure and some physico-chemical properties of beef, doi: https://doi.org/10.1016/S0309-1740(98)00094-1, Meat Sci., 51, 35-42 (1999)

Higuera, O.A., W. Torres y otros cuatro autores, Effect of pulsed ultrasound on the physicochemical characteristics and emulsifying properties of squid (Dosidicus gigas) mantle proteins, doi: https://doi.org/10.1016/j.ultsonch.2017.01.008, Ultrasonic Sonochemistry, 38, 829-834 (2017)

Higuera, O.A., C.L. Del Toro, S. Ruiz, E. Márquez, Effects of high-energy ultrasound on the functional properties of proteins, doi: http://dx.doi.org/10.1016/j.ultsonch.2016.02.007, Ultrasonics Sonochemistry, 31, 558-562 (2016)

Hu Y.Q, H.X. Yu, K.C. Dong, S.B. Yang, X.Q. Ye y S.G. Chen, Analysis of the tenderisation of jumbo squid (Dosidicus gigas) meat by ultrasonic treatment using response surface methodology, doi: https://doi.org/10.1016/j.foodchem.2014.01.085, Food Chem. 160, 19-225 (2014)

Jayasooriya, S. D., P. Torley, B.R. D'arcy y B.R. Bhandari, Effect of high power ultrasound and ageing on the physical properties of bovine Semitendinosus and Longissimus muscles, doi: https://doi.org/10.1016/j.meatsci.2006.09.010, Meat Science, 75(4), 628-639 (2007)

Kang, D, Y. Zou, Y. Cheng, L. Xing, G. Zhou y W. Zhang, Effects of power ultrasound on oxidation and structure of beef proteins during curing processing, doi : https://doi.org/10.1016/j.ultsonch.2016.04.024, Ultrasonics Sonochemistry,33, 47$53(2016)$

$\mathrm{Li}, \mathrm{Q}$., Y. Wang y otros ocho autores, Effects of age on slaughter performance and meat quality of Binlangjang male buffalo, doi: https://doi.org/10.1016/j.sjbs.2017.10.001, Saudi Journal of Biological Sciences, 25, 248-252 (2018)

López, J.R., Perspectivas de la crianza del búfalo de agua (Bubalus bubalis) en la Amazonía Ecuatoriana, ISSN: 13908049 Revista Amazónica: Ciencia y Tecnología, 2(1), 19-29 (2013)

O'Sullivan, J., B. Murray, C. Flynn e I. Norton, The effect of ultrasound treatment on the structural, physical and emulsifying properties of animal and vegetable proteins, doi: https://doi.org/10.1016/j.foodhyd.2015.02.009, Food Hydrocolloids, 53, 141-154 (2016)

Robles, L.E y L.A. Ochoa, Ultrasonido y sus aplicaciones en el procesamiento de alimentos, ISSN: 1665-0204, Rev. Iber. Tecnología Postcosecha, 13(2),109-122 (2012)

Rosenthal, A.J., Food Texture: Measurement and Perception: Measurement and Perception, $2^{\mathrm{a}}$ Ed., 183-187, Aspen Publishers Inc, Philadelphia, USA (1999)

Ruiz de Huidobro, F., E. Miguel, B. Blázquez y E. Onega, A comparison between two methods (Warner-Bratzler and texture profile analysis) for testing either raw meat or cooked meat, doi: https://doi.org/10.1016/j.meatsci.2004.09.008, Meat Science, 69(3), 527-536 (2005)

Sachindra, N.M., P.Z. Sakhare, K.P. Yashoda y D. Narasimha, Microbial profile of buffalo sausage during processing and storage, doi: https://doi.org/10.1016/j.foodcont.2003.11.002, Food Control, 16, 31-35 (2005)

Sikes, A.L, R. Mawson, J. Stark y Robyn Warne, Quality properties of pre- and post-rigor beef muscle after interventions with high frequency ultrasound, doi: https://doi.org/10.1016/j.ultsonch.2014.03.008, Ultrasonics Sonochemistry, 212138$2143(2014)$

Singh, V., N. Guizani, A. Al-Alawi, M. Claereboudt y M.S. Rahman, Instrumental texture profile analysis (TPA) of date fruits as a function of its physico-chemical properties, doi: https://doi.org/10.1016/j.indcrop.2013.08.039, Industrial Crops and Products, 50,866-873 (2013)

Sorapukdee, S., C. Kongtasorn, S. Benjakul y W. Visessanguan, Influences of muscle composition and structure of pork from different breeds on stability and textural properties of cooked meat emulsion, doi: https://doi.org/10.1016/j.meatsci.2006.02.017, Food Chemistry, 138 1892-1901 (2013)

Thomas, R., A. Anjaneyulu y R. Kondaiah, Quality and shelf life evaluation of emulsion and restructured buffalo meat nuggets at cold storage, doi: https://doi.org/10.1016/j.meatsci.2005.03.022, Meat Sci, 72(3), 373-379 (2006) 
Torres, R., E.J. Montes, O.A. Pérez y R.D. Andrade, Influencia del Color y Estados de Madurez sobre la Textura de Frutas Tropicales (Mango, Papaya y Plátano), doi: http://dx.doi.org/10.4067/S0718-07642015000300008, Información Tecnológica, 26(3), 47-52 (2015)

Uriyapongson, S., 2013. Buffalo and buffalo meat in Thailand. Buffalo Bull. 32 (Special Issue 1), $329-332$ (2013)

Warner, R.D., C.K. McDonnell y otros seis autores, Systematic review of emerging and innovative technologies for meat tenderisation, doi: http://dx.doi.org/10.1016/j.meatsci.2017.04.241, Meat Science 132, 72-89 (2017)

Zhang, Q.T., Z.C. Tu y otros cinco autores, Influence of ultrasonic treatment on the structure and emulsifying properties of peanut protein isolate, doi: https://doi.org/10.1016/j.fbp.2013.07.006, Food Bioprod., Process, 92(1)), 30-37 (2014)

Zou, Y., K. Zhang y otros cuatro autores, Rapid tenderizing of goose breast muscle based on actomyosin dissociation by lowfrequency ultrasonication, doi: https://doi.org/10.1016/j.procbio.2017.11.010, Process Biochemistry, 65, 115-122 (2018) 
